\title{
Implementation of Obstetric Telehealth During COVID-19 and Beyond
}

\author{
Kimberly Fryer ${ }^{1}\left[\right.$ [ $\cdot$ Arlin Delgado $^{1} \cdot$ Tara Foti $^{2} \cdot$ Chinyere N. Reid ${ }^{2} \cdot$ Jennifer Marshall $^{3}$
}

Published online: 20 June 2020

(c) Springer Science+Business Media, LLC, part of Springer Nature 2020

\begin{abstract}
Purpose The purpose of this article is to illustrate and discuss the impact the 2019 novel Coronavirus (COVID-19) pandemic on the delivery of obstetric care, including a discussion on the preexisting barriers, prenatal framework and need for transition to telehealth.

Description The COVID-19 was first detected in China in December of 2019 and by March 2020 spread to the United States. As this virus has been associated with severe illness, it poses a threat to vulnerable populations_including pregnant women. The obstetric population already faces multiple barriers to receiving quality healthcare due to personal, environmental and economic barriers, now challenged with the additional risks of COVID-19 exposure and limited care in times much defined by social distancing.

Assessment The current prenatal care framework requires patients to attend multiple in-office prenatal visits that can exponentially multiply depending on maternal and fetal comorbidities. To decrease the rate of transmission of the COVID19 and limit exposure to patients, providers in Hillsborough County, Florida (and nationwide) are rapidly transitioning to telehealth. The use of a virtual care model allows providers to reduce in-person visits and incorporate virtual visits into the schedule of prenatal care.

Conclusion Due to the COVID-19 pandemic, implementation of telehealth and telehealth have become crucial to ensure the safe and effective delivery of obstetric care. This implementation is one that will continue to require attention to planning, procedures and processes, and thoughtful evaluation to ensure the sustainability of telehealth and telehealth post COVID-19 pandemic.
\end{abstract}

Keywords Prenatal care $\cdot$ Telemedicine $\cdot$ Telehealth $\cdot$ Obstetrics $\cdot$ COVID-19

\section{Significance}

The public health system in the United States must rise to the challenge to meet this public health crisis and innovate new ways to provide care, including prenatal care to vulnerable women.

Kimberly Fryer

kfryer@usf.edu

1 Department of Obstetrics and Gynecology, Morsani College of Medicine, University of South Florida, 2 Tampa General Circle, 6th Floor, Tampa, FL 33606, USA

2 Chiles Center, College of Public Health, University of South Florida, 13201Bruce B Downs Blvd, Tampa, FL 33612, USA

3 Sunshine Education and Research Center, Chiles Center College of Public Health, University of South Florida, 13201 Bruce B Downs Blvd, Tampa, FL 33612, USA

\section{Coronavirus Outbreak}

The 2019-novel Coronavirus (COVID-19), a severe acute respiratory syndrome caused by coronavirus 2 , was first detected in Wuhan, China in December 2019, (Luo et al 2020; Zhu et al 2019) subsequently spreading around the world. By January, the first case of COVID-19 was reported in the United States in Washington State (Centers for Disease Control and Prevention [CDC] 2020a; CDC 2020b; Holshue et al 2020), and by March, COVID-19 had reached Florida (Florida Department of Health [FDOH] 2020). By January 27, a State of Emergency was declared by the U.S. Health and Human Services (DHHS) (U.S. DHHS 2020a, b). On March 11, 2020 the disease outbreak was declared a pandemic by the World Health Organization (CDC 2020c). Since then, Florida has ranked 7 th in the nation with its number of confirmed cases surpassing 16,000 (FDOH 
2020), behind other epicenters such as New York, California, and Louisiana.

Much is still unknown about vulnerabilities, treatment, and long-term effects of the virus. As a vulnerable group, pregnant women have a higher risk of developing complications from other viral respiratory infections, though it is still unknown whether they have a higher vulnerability to the COVID-19 virus (Rasmussen et al. 2020). Other than a single, small retrospective study of pregnant women who developed COVID-19 pneumonia in their third trimester and showed no vertical transmission (Chen et al 2020), there is presently no evidence of maternal-fetal vertical transmission in the literature.

There are over 220,000 births in Florida each year, with an average of 17,000 in Hillsborough County alone (FDOH 2020). During this time of uncertainty, pregnant women need prenatal care more than ever, in order to monitor their health and well-being, answer their questions, and check on the progress of their pregnancy. Disasters amplify vulnerabilities and barriers to care; this pandemic has caused numerous challenges that compound barriers.

\section{Existing Prenatal Care Delivery System}

The current model of prenatal care is built from expert opinion and tradition rather than based on evidence. Prenatal care was first created in the early 1900s to decrease and prevent infant low birth weight and eclampsia (Alexander and Kotelchuck 2001). In the low-risk patient with no comorbidities, visits are recommended to occur every 4 weeks until 28 weeks gestational age, subsequently becoming every 2 weeks until 36 weeks gestational age, and then weekly until delivery. Through this prenatal care system, women often receive additional visits for ultrasounds and genetic screening (ACOG 2016). If a woman is considered high risk for complications due to comorbidities such as chronic hypertension or diabetes, these 14 visits multiply exponentially for increased monitoring of the fetus, as well as the mother.

\section{Barriers to Prenatal Care}

Many models have been developed to identify and explain barriers to adequate prenatal care. We have created a combined theoretical framework for prenatal access to care (Fig. 1), based on the work of Khan and Bhardwaj (1994) and Phillippi and Roman (2013) to synthesize the factors
Fig. 1 Combined theoretical framework for access to care adapted from Phillipi and Khan (Phillippi 2013; Khan 1994)

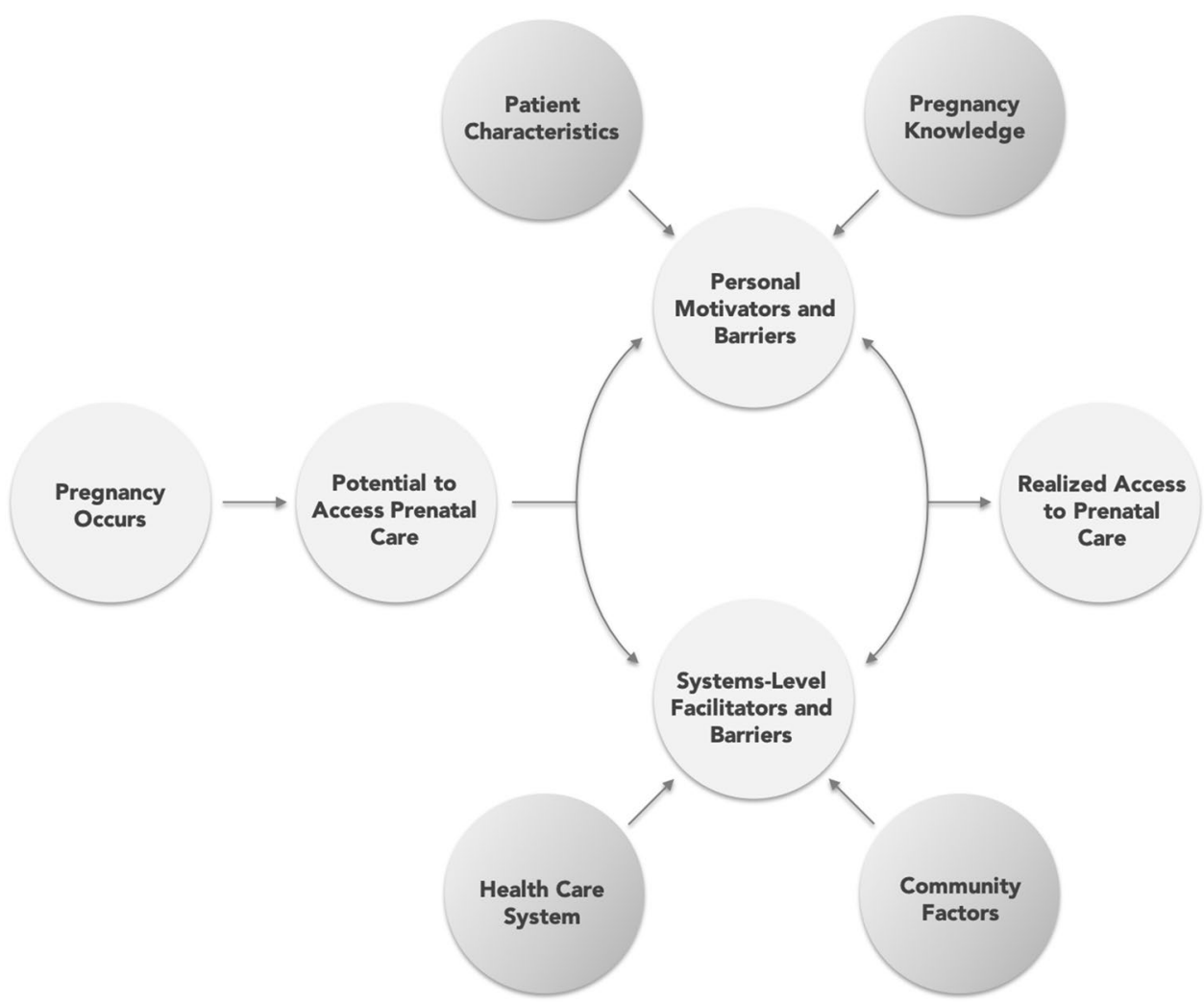


affecting access to prenatal care. We divided access to care into personal- and systems-level motivators and barriers. Personal motivators and barriers include factors such as pregnancy knowledge, attitudes about pregnancy and social support. From a systems level, the health care system itself can be either a facilitator or a barrier to care as well as the community (e.g. access to transportation).

\section{Personal Motivators and Barriers}

Personal barriers can be expanded to include individual or psychological factors, patient knowledge and attitudes, and socioeconomics. There are a number of individual or psychological barriers to obtaining prenatal care, such as not feeling well or having low energy or fatigue (Torres 2016); depression (Daniels Noe and Mayberry 2006); life stress, chaos or crises (Heaman et al 2015; Johnson et al 2011; Mazul Salm Ward and Ngui 2017); and personal drug or alcohol addictions (Heaman et al 2015; Roberts and Pies 2011; Torres 2016). Knowledge and attitudes barriers to prenatal care, such as a general lack of awareness of the signs and symptoms of pregnancy (Daniels et al. 2006). Women may also delay prenatal care while considering abortion (Johnson et al 2011; Torres 2016), out of fear of pregnancy disclosure (Braveman et al 2000), or due to negative emotional responses to pregnancy (Daniels et al 2006; Torres, 2016). Also, this may include not knowing or believing that prenatal care is important (Heaman et al. 2015; Torres 2016); not knowing when to start prenatal care (Braveman et al 2000); or having a negative attitude toward prenatal care (Mazul et al. 2017).

Additionally, social and relationship factors may also function as prenatal care barriers. This may include being unmarried or single, having relationship problems, experiencing abuse or intimate partner violence or having an unreliable partner (Cha et al. 2014; Heaman et al 2015). Women who want their partner to attend prenatal care appointments with them may delay appointments until they can do so (Daniels et al 2006). Finally, some women have reported having a person or people in their lives who prevented them from attending prenatal care appointments (Torres 2016).

\section{Systems-Level Motivators and Barriers}

Systems-level barriers include both the health care system as well as the community at large, that also plays a role in prenatal care attainment. In the healthcare system, these may include poor past experiences or relationships with providers; dissatisfaction or perceived low-quality care; feelings that providers do not listen, take concerns seriously or spend enough time with them; and providers with negative personality characteristics resulting in late or inadequate prenatal care (Heaman et al 2015; Mazul et al 2017; Roberts and Pies 2011; Torres 2016). Factors such as problems with scheduling timely appointments, clinic location or hours that are inconvenient, shortages of prenatal care providers and cost may function as barriers to prenatal care (Heaman et al 2015; Roberts and Pies 2011; Torres 2016).

Community factors include living in poverty, receiving public assistance, unemployment and homelessness (Ayoola et al 2010; Heaman et al 2013; Johnson et al 2011; Mazul et al 2017). Employed individuals with low paying jobs may be reticent to take time off work for prenatal care appointments (Heaman et al 2015). Women of color, migrant women, and women with low English language proficiency are all at increased risk for receiving suboptimal prenatal care (Ayoola et al 2010; Beckmann Buford and Witt 2000; Heaman et al 2013). Additionally, childcare and transportation are barriers to prenatal care (Heaman et al 2015; Mazul et al 2017; Torres 2016).

Many of these barriers are prevalent in Hillsborough County, which is a racially/ethnically diverse county $(25 \%$ minority population, $17 \%$ foreign-born), with both urban and rural communities, $12 \%$ of those over age 25 with less than a high school education, and $15 \%$ of residents living in poverty (US Census Quickfacts Bureau, 2020). However, 85\% of the population from 2014-2018 had an internet subscription and $92 \%$ reported that they had a computer in the home (US Census Quickfacts, Bureau 2020). Another study by the authors of this paper found that barriers to prenatal care among diverse patients were primarily related to community/social conditions, healthcare system factors, and personal factors. The most frequent barriers were that women couldn't get an appointment when they wanted or needed one, they didn't have insurance, or that they were not aware of the pregnancy (Unpublished data).

\section{Implications of the COVID-19 Pandemic}

In addition to these barriers identified, COVID-19 now presents new specific barriers to prevent full prenatal care participation. First, women may avoid care due to infectious concerns or guidance to participate in social distancing or self-quarantine. The practice of social distancing has led to school and daycare closures that potentially add to childcare and transportation barriers. Additionally, women may be balancing working from home with childcare needs or assisting with remote education of older children. These competing demands and drastically limited social interaction, at least in-person, may also exacerbate mental health or stress issues during pregnancy. Healthcare-related policies and practice issues in response to new social practices may impact prenatal care by limiting the attendance of support systems to attend appointments, clinics may face staffing shortages and lack of personal protective equipment. 
Finally, changes in prenatal care itself, may serve as a barrier to care. Suggested reductions or changes to prenatal care and ultrasound visits may be confusing, poorly communicated or evoke fear. Women may avoid prenatal care due to infectious symptoms, isolation, or treatment for suspected or confirmed COVID-19. In an effort to remotely provide prenatal care, telehealth visits may be inevitable. Barriers to rapid implementation of prenatal telehealth visits may include a lack of technology, high start-up costs, the need for HIPAA compliance, integration with existing electronic medical record systems, and provider malpractice insurance coverage (Kaiser Family Foundation 2020). Additionally, while Medicaid has temporarily expanded coverage for telehealth visits (Centers for Medicaid Services [CMS], 2020), there is a lack of coverage, or clarity surrounding coverage of telehealth visits by other insurance providers (Kaiser Family Foundation 2020).

\section{Obstetric Telehealth Model}

Due to the desire to prevent infections in the community and continue obstetric care, many clinics are moving towards telehealth. Telehealth can encompass many different modalities including remote monitoring or remote reading of images, but in the time of COVID-19, the main growth of telehealth has come in the form of virtual visits (Abelson 2020; ACOG 2020). Changes in reimbursement models from multiple payers, including Medicare and Medicaid (CMS 2020), have incentivized multiple health systems in the Tampa Bay area to rapidly implement virtual visits (BaycareAnywhere 2020; Expert Virtual Care 2020 2020; Virtual Care 2020). Additionally, need for a HIPPA compliant platform has also been temporarily waived in the pandemic (U.S. DHHS 2020a, b). Below, we will discuss our clinical protocol and its rapid development and launch.

\section{Essential Elements of a Telehealth Visit}

In order to be classified as a telehealth visit by CMS rules, the visit must include two-way audio and video communication. Therefore, the first element needed is a virtual platform for video communication. This platform needs to be HIPPA compliant, and multiple platforms exist (Coronavirus: How telehealth can help 2020; Williams 2020; Zoom 2020). Some platforms incorporate into existing electronic medical record systems, and others are stand alone.

\section{Protocol Development}

The next major element is a uniform obstetric protocol for all low risk patients in a practice. In the rapid transition to telehealth in obstetrics, we have adapted a virtual care model (OB Nest) with robust clinical trials developed in Minnesota (de Mooij et al 2018) to develop a new protocol to reduce in-person visits and incorporate telehealth visits into the schedule of prenatal care. OB Nest is a combined virtual prenatal care protocol that combines eight in-person visits with six virtual nursing visits, as well as an asynchronous online portal for questions and education, and an online community for patients moderated by nurses. Participants were provided with blood pressure cuffs and fetal dopplers. In a randomized comparison of OB Nest and standard care, they found that participants in OB Nest had higher patient satisfaction and lower stress (Butler et al. 2019).

In order to accomplish this locally, we have adapted the OB Nest protocol (de Mooij et al 2018). Patients presenting to the clinic when physical exams, ultrasounds or labs are needed and using virtual visits appropriate. Patients have also been recommended to purchase blood pressure cuffs, scales, and optionally, fetal Dopplers to monitor vital signs from home. To assist patients with financial concerns, we have collected generous donations from community organizations, as well as applied for assistance programs to reduce financial burdens. Additionally, in-person visits may be added for urgent concerns/issues. We do not have an online community, and we have decreased the number of in-person visits to a maximum of six to reduce the exposure to possible infection. This protocol (Table 1) was rapidly developed in consultation with national leaders in virtual obstetric care, including from the University of Utah and Mayo Clinic; and consultation with local leaders in midwifery, Maternal Fetal Medicine specialists, and specialists in Academic Obstetrics and Gynecology. We also relied on patient education materials and protocols created at the University of Michigan for the COVID19 response (University of Michigan 2020). Due to the immediate need, this protocol was rapidly developed and

Table 1 Example in-person and virtual visit schedule

\begin{tabular}{ll}
\hline Prenatal care visit schedule & \\
\hline Nursing virtual visit & $6-10$ weeks \\
New OB visit & $10-14$ weeks \\
Virtual visit & $15-19$ weeks \\
In person visit & $20-22$ weeks \\
Virtual visit & $23-26$ weeks \\
In person visit & $27-28$ weeks \\
Virtual visit & $29-34$ weeks \\
In person visit & $35-36$ weeks \\
Virtual visit & $37-38$ weeks \\
In person visit & $39-40$ weeks \\
In person visit & $40-41$ weeks \\
Virtual visit & 2 weeks postpartum \\
Virtual visit & 6 weeks postpartum \\
\hline
\end{tabular}


implemented in less than two weeks in the obstetric clinic in contrast to a typical timeline of months to years for making major institutional changes.

\section{Implementation Monitoring and Evaluation}

\section{Components and Constructs}

Implementation and evaluation of this rapid transition to obstetric telehealth requires attention to structure, processes and outcomes (Donabedian 1968). Structural considerations include financing, technology, staffing, and other resources that affect availability and access to care. Communication, bioethics, training, and logistical processes should be established, with measures in place for delivery of services. The desired outcomes of obstetric care (services provided/care received, patient and provider satisfaction, healthy pregnancy) continue to be monitored, in comparison to traditional care models, with attention to how continuity of care was maintained in this transition. Pflugeisen and Mou (2017) measured satisfaction with virtual and traditional prenatal care in five domains-scheduling, provider, personal, care assessment, and technology, and suggested assessing patient familiarity and comfort with different videoconference applications, variation in quality of wireless connectivity on the patient or provider end, or technology platform. They also recognized that some patients (e.g. first-time pregnancies) may prefer in-person care.

Chang's (2015) review of multiple evaluative frameworks for telehealth recognized these factors reflected across studies, including Bashshur et al.'s (2011) "taxonomy of telemedicine," which includes measurement of functionality, applications, and technology, and Nepal et al.'s (2014) six dimensions of design, implementation, and evaluation of telehealth (health domains, health services, technologies, communication infrastructure, environmental settings, and socioeconomic analysis). Ultimately, Chang's (2015) comprehensive evaluation framework encompasses nearly 30 human, system and environment components in six domains (service providers and patients, organizational and technology, society and policies) leading to three outcomes: cost effectiveness, quality of care, and patient satisfaction.

This project uses the Consolidated Framework for Implementation Research (CFIR), developed by Damschroder et al. (2009), which aids in analyzing and organizing findings from implementation research. CFIR Constructs include intervention characteristics, outer setting (e.g. patient needs, expectations, resources), inner setting (e.g., organizational culture, buy-in, leadership), characteristics of the individuals involved, and the process of implementation (e.g., planning, evaluation).

\section{Evaluation Approach}

Ongoing evaluation tests the feasibility and acceptability of each step in the process and component of care from the perspectives of administrators, health care providers, and patients for quick adjustments. Several approaches exist to measuring processes and outcomes. Traditional methods include analysis of patient data connecting activities to outcomes or measuring various dimensions of patient satisfaction (Pflugeisen and Mou 2017). Alternatively, Chang (2015) suggested that an evaluation should focus on identifying and addressing barriers to implementation in five categories: behavioral (health care providers' attitudes), organizational (leadership, communication), technical capacity and infrastructure, economic, and legal.

Another approach to evaluation is developmental. The telehealth maturity model (TMMM) is an iterative top-down approach that emphasizes the processes within micro, meso, and macrolevels of a telehealth service development (Van Dyk and Schulte 2013). A quality improvement and innovation development approach, like the framework developed by the Institute for Healthcare Improvement (IHI) (Martin and Mate 2018), provides the architecture, processes, and management system for developing and trying new processes at the micro scale for a brief period before fully implementing.

Process mapping and journey mapping (McCarthy et al 2016; Trebble et al 2010) can highlight gaps and areas for improvement from the provider or patient perspective from beginning to end. Telehealth may work differently for some patients or providers than others, and journey mapping establishes personas as patient or provider subgroups based on their desires/preferences, and expectations for obstetric care.

To evaluate our implemented prenatal model, we have adapted the CFIR rapid cycle evaluation approach (Damshroeder et al. 2009; Keith et al. 2017). This framework allows us to examine the culture of the clinic as well as attitudes of both providers and patients. Using this framework, we created patient and provider anonymous online surveys regarding their own experiences, perceived advantages and challenges with telehealth. Furthermore, we plan to phone interview both patients and providers for further in-depth qualitative information in the coming months.

\section{Looking to the Future Post COVID-19}

Certainly COVID-19 has presented countless challenges to health, healthcare access and delivery in obstetric medicine. The pandemic has forced providers and systems to consider access to care in a new way as few patients are physically accessing care due to social distancing policies and fear of exposure to the virus. Escalating cases have also started to 
strain healthcare system capacities-facilities, staff, and supplies. Thus, telehealth is being quickly implemented across health and social services in order to meet community needs and to promote continuity of care. Attention to planning, documentation of policies, procedures and processes, and ongoing evaluation will facilitate post-COVID-19 implementation, improvement and sustainability of telehealth programs that may improve access and utilization of prenatal care across the board. Our long-term goal is to reduce barriers to prenatal care such as lack of transportation, childcare and reduction of long wait times requiring missed work or schooling by implementing telemedicine. We acknowledge that this model of care may not be ideal for all patients, but it remains a viable option for some women who may prefer the convenience of fewer in-person visits and serves as a prenatal care model option (Peahl et al. 2020a; b).

In the first few weeks of implementation, our program has made available a survey to providers and patients, using CFIR constructs to measure implementation, utility, feasibility, and satisfaction by addressing the intervention as well as setting in which it is applied. With this feedback, we are then able to further assess and addend the current protocol first implemented out of necessity with the goal of creating a sustainable model to reduce barriers post COVID-19 prenatal care.

\section{References}

Abelson, R. (2020). Doctors and Patients Turn to Telehealth in the Coronavirus Outbreak. The New York Times. Retrieved from https ://www.nytimes.com/2020/03/11/health/telehealth-coronaviru s.html?searchResultPosition $=2$.

American College of Obstetricians and Gynecologists. (2016). Practice Bulletin Number 167. Screening for fetal aneuploidy. Obstetrics and Gynecology, 127(5), 123-137. https://doi.org/10.1097/ AOG.0000000000001406.

American College of Obstetricians and Gynecologists. (2020). Committee Opinion Number 798 implementing telehealth in practice. Obstetrics and Gynecology., 135(2), e73-e79.

Alexander, G. R., \& Kotelchuck, M. (2001). Assessing the role and effectiveness of prenatal care: History, challenges, and directions for future research. Public Health Reports, 116(4), 306-316. https ://doi.org/10.1016/S0033-3549(04)50052-3.

Ayoola, A. B., Nettleman, M. D., Stommel, M., \& Canady, R. B. (2010). Time of pregnancy recognition and prenatal care use: A population-based study in the United States. Birth, 37(1), 37-43. https://doi.org/10.1111/j.1523-536X.2009.00376.x.

BaycareAnywhere. (2020). Baycare Health Organization. Retrieved from https://baycare.org/baycareanywhere.

Bashshur, R., Shannon, G., Krupinski, E., \& Grigsby, J. (2011). The taxonomy of telehealth. Telehealth and e-Health, 17(6), 484-494. https://doi.org/10.1089/tmj.2011.0103.

Beckmann, C. A., Buford, T. A., \& Witt, J. B. (2000). Perceived barriers to prenatal care services. MCN American Journal of Maternal/ Child Nursing, 25(1), 43-46. https://doi.org/10.1097/00005721200001000-00009.

Braveman, P., Marchi, K., Egerter, S., Pearl, M., \& Neuhaus, J. (2000). Barriers to timely prenatal care among women with insurance: The importance of prepregnancy factors. Obstetrics \& Gynecology, 95(6), 874-880. https://doi.org/10.1016/s0029 -7844(00)00780-8.

Butler Tobah, Y. S., LeBlanc, A., Branda, M. E., Inselman, J. W., Morris, M. A., Ridgeway, J. L., et al. (2019). Randomized comparison of a reduced-visit prenatal care model enhanced with remote monitoring. American Journal of Obstetrics and Gynecology. https://doi.org/10.1016/j.ajog.2019.06.034.

Center for Medicare and Medicaid Services. (2020). Medicare telehealth health care provider fact sheet. Retrieved from https:// www.cms.gov/newsroom/fact-sheets/medicare-telehealth-healt h-care-provider-fact-sheet.

Centers for Disease Control and Prevention. (2020a, April). Cases in U.S. Retrieved from https://www.cdc.gov/coronavirus/2019-ncov/ cases-updates/cases-in-us.html\#cumulative.

Centers for Disease Control and Prevention. (2020b, April). How Coronavirus Spreads. Retrieved from https://www.cdc.gov/coronaviru s/2019-ncov/prevent-getting-sick/how-covid-spreads.html?CDC_ AA_refVal=https $\% 3 \mathrm{~A} \% 2 \mathrm{~F} \% 2 \mathrm{Fwww} . \mathrm{cdc}$.gov\%2Fcoronavi rus\%2F2019-ncov\%2Fabout\%2Findex.html.

Centers for Disease Control and Prevention. (2020c). Situation Summary. Retrieved from https://www.cdc.gov/coronaviru s/2019-ncov/cases-updates/summary.html?CDC_AA_refVa $1=$ https $\% 3 \mathrm{~A} \% 2 \mathrm{~F} \% 2 \mathrm{Fwww} . c d c . g o v \% 2 \mathrm{Fcoronavirus} \% 2 \mathrm{~F} 201$ 9-ncov\%2Fsummary.html\#emergence.

Cha, S., \& Masho, S. W. (2014). Intimate partner violence and utilization of prenatal care in the United States. Journal of Interpersonal Violence, 29(5), 911-927. https://doi.org/10.1177/08862 60513505711.

Chang, H. (2015). Evaluation framework for telehealth using the logical framework approach and a fishbone diagram. Healthcare Informatics Research, 21(4), 230-238. https://doi.org/10.4258/ hir.2015.21.4.230.

Chen, H., Guo, J., Wang, C., Luo, F., Yu, X., Zhang, W., et al. (2020). Clinical characteristics and intrauterine vertical transmission potential of COVID-19 infection in nine pregnant women: A retrospective review of medical records. The Lancet, 395(10226), 809-815. https://doi.org/10.1016/S0140-6736(20)30360-3.

Coronavirus: How telehealth can help. (2020). Amwell. Retrieved from https://business.amwell.com/

Damschroder, L. J., Aron, D. C., Keith, R. E., Kirsh, S. R., Alexander, J. A., \& Lowery, J. C. (2009). Fostering implementation of health services research findings into practice: A consolidated framework for advancing implementation science. Implementation Science, 4, 50. https://doi.org/10.1186/1748-5908-4-50.

Daniels, P., Noe, G. F., \& Mayberry, R. (2006). Barriers to prenatal care among black women of low socioeconomic status. American Journal of Health Behavior, 30(2), 188-198. https://doi. org/10.5993/AJHB.30.2.8.

de Mooij, M. J. M., Hodny, R. L., O’Neil, D. A., Gardner, M. R., Beaver, M., Brown, A. T., et al. (2018). OB Nest: Reimagining low-risk prenatal care. Mayo Clinic Proceedings. https://doi. org/10.1016/j.mayocp.2018.01.022.

Donabedian, A. (1968). The evaluation of medical care programs. Bulletin of the New York Academy of Medicine, 44(2), 117-124.

Expert Virtual Care. (2020). Advent Health. Retrieved from https:// www.adventhealth.com/eCare.

Florida Department of Health. (n.d.). Florida Birth Query System. In FLHealthCHARTS. Retrieved from: https://www.flhealthcharts. com/FLQUERY/Birth/BirthRpt.aspx.

Florida Department of Health (2020, April). Florida's COVID-19 data and map dashboard. Retrieved April 9, 2020, from https://exper ience.arcgis.com/experience/96dd742462124fa0b38ddedb9b25e4 29.

Heaman, M., Bayrampour, H., Kingston, D., Blondel, B., Gissler, M., Roth, C., et al. (2013). Migrant women's utilization of prenatal 
care: A systematic review. Maternal and Child Health Journal, 17(5), 816-836. https://doi.org/10.1007/s10995-012-1058-z.

Heaman, M. I., Sword, W., Elliott, L., Helewa, M. E., Morris, H., Gregory, P., et al. (2015). Barriers and facilitators related to use of prenatal care by inner-city women: Perceptions of health care providers. Biomed Central Pregnancy and Childbirth. https://doi. org/10.1186/s12884-015-0431-5.

Holshue, M. L., DeBolt, C., Lindquist, S., Lofy, K. H., Wiesman, J., Bruce, H., et al. (2020). First case of 2019 novel coronavirus in the United States. New England Journal of Medicine, 382, 929-936. https://doi.org/10.1056/NEJMoa2001191.

Johnson, A. A., Wesley, B. D., El-Khorazaty, M. N., Utter, J. M., Bhaskar, B., Hatcher, B. J., et al. (2011). African American and Latino patient versus provider perceptions of determinants $\mathrm{f}$ prenatal care utilization. Maternal Child Health Journal, 15(Suppl 1), S27-34. https://doi.org/10.1007/s10995-011-0864-z.

Kaiser Family Foundation. (2020). Novel coronavirus "Covid-19": Special considerations for pregnant women. Retrieved from https ://www.kff.org/womens-health-policy/issue-brief/novel-coron avirus-covid-19-special-considerations-for-pregnant-women/.

Keith, R. E., Crosson, J. C., O’Malley, A. S., Cromp, D. A., \& Taylor, E. F. (2017). Using the Consolidated Framework for Implementation Research (CFIR) to produce actionable findings: A rapid-cycle evaluation approach to improving implementation. Implementation Science, 12(1), 1-12. https://doi.org/10.1186/ s13012-017-0550-7.

Khan, A. A., \& Bhardwaj, S. M. (1994). Access to health care: A conceptual framework and its relevance to health care planning. Evaluation \& The Health Professions, 17(1), 60-76. https://doi. org/10.1177/016327879401700104.

Luo, C., Yao, L., Zhang, L., Yao, M., Chen, X., Wang, Q., et al. (2020). Possible transmission of severe acute respiratory syndrome coronavirus 2 (SARS-CoV-2) in a public bath center in Huai' an, Jiangsu Province, China. Journal of American Medical Association Network Open, 3(3), e204583. https://doi.org/10.1001/jaman etworkopen.2020.4583.

Martin L. A. \& Mate, K. (2018). IHI innovation system. IHI White Paper; Boston, Massachusetts: Institute for Healthcare Improvement. Retrieved from https://www.ihi.org.

Mazul, M. C., Salm Ward, T. C., \& Ngui, E. M. (2017). Anatomy of good prenatal care: Perspectives of low-income African American women on barriers and facilitators to prenatal care. Journal of Racial Ethnic Health Disparities, 4(1), 79-86. https://doi. org/10.1007/s40615-015-0204-X.

McCarthy, S., O'Raghallaigh, P., Woodworth, S., Lim, Y. L., Kenny, L. C., \& Adam, F. (2016). An integrated patient journey mapping tool for embedding quality in healthcare service reform. Journal of Decision Systems, 25(sup1), 354-368. https://doi. org/10.1080/12460125.2016.1187394.

Nepal, S., Li, J., Jang-Jaccard, J., \& Alem, L. (2014). A framework for telehealth program evaluation. Telehealth and e-Health, 20(4), 393-404.

Peahl, A. F., Gourevitch, R. A., Luo, E. M., Fryer, K. E., Moniz, M. H., Dalton, V. K., et al. (2020). Right-sizing prenatal care to meet patients' needs and improve maternity care value. Obstetrics \& Gynecology, 135(5), 1027-1037. https://doi.org/10.1097/ aog.0000000000003820.

Peahl, A. F., Novara, A., Heisler, M., Dalton, V. K., Moniz, M. H., \& Smith, R. D. (2020). Patient preferences for prenatal and postpartum care delivery. Obstetrics \& Gynecology, 135(5), 1038-1046. https://doi.org/10.1097/aog.0000000000003731.

Pflugeisen, B. M., \& Mou, J. (2017). Patient satisfaction with virtual obstetric care. Maternal and Child Health Journal, 21(7), 15441551. https://doi.org/10.1007/s10995-017-2284-1.
Phillippi, J. C., \& Roman, M. W. (2013). The motivation-facilitation theory of prenatal care access. Journal of Midwifery \& Women's Health, 58(5), 509-515. https://doi.org/10.1111/jmwh.12041.

Rasmussen, S. A., Smulian, J. C., Lednicky, J. A., Wen, T. S., \& Jamieson, D. J. (2020). Coronavirus disease 2019 (COVID-19) and pregnancy: What obstetricians need to know. American Journal of Obstetrics \& Gynecology. https://doi.org/10.1016/j. ajog.2020.02.017.

Roberts, S. C. M., \& Pies, C. (2011). Complex calculations: How drug use during pregnancy becomes a barrier to prenatal care. Maternal Child Health Journal, 15(3), 333-341. https://doi.org/10.1007/ s10995-010-0594-7.

Torres, R. (2016). Access barriers to prenatal care in emerging adult Latinas. Hispanic Health Care International, 14(1), 10-16. https ://doi.org/10.1177/1540415316631504.

Trebble, T. M., Hansi, N., Hydes, T., Smith, M. A., \& Baker, M. (2010). Process mapping the patient journey: An introduction. Biomedical Journal, 341, c4078. https://doi.org/10.1136/bmj.c4078.

U.S. Census Bureau (2020) . U.S. Quickfacts. Retrieved from https:// www.census.gov/quickfacts/fact/table/hillsboroughcountyflorida /RHI225218\#RHI225218.

U.S. Department of Health and Human Services. (2020a, January). Determination that a Public Health Emergency Exists. Retrieved from https://www.phe.gov/emergency/news/healthactions/phe/ Pages/2019-nCoV.aspx.

U.S. Department of Health and Human Services. (2020b, March). COVID-19 \& HIPAA Bulletin Limited Waiver of HIPAA sanctions and penalties during a nationwide public health emergency. Retrieved from https://www.hhs.gov/sites/default/files/hipaa-andcovid-19-limited-hipaa-waiver-bulletin-508.pdf.

University of Michigan. (2020). Prenatal care during the COVID-19 pandemic: Prenatal patient resources. Retrieved from https:// medicine.umich.edu/dept/obgyn/patient-care-services/prenatalcare-during-covid-19-pandemic-prenatal-patient-resources

Van Dyk, L. \& Schutte, C. (2013, December 13th). The Telehealth service maturity model: A framework for the measurement and improvement of telehealth services, telehealth, In R. Madhavan \& S. Khalid, (Eds.), IntechOpen. Retrieved from https://www. intechopen.com/books/telehealth/the-telehealth-service-matur ity-model-a-framework-for-the-measurement-and-improvemen t-of-telemedic

Virtual Care. (2020). Tampa General Hospital Organization. Retrieved from https://www.tgh.org/services/virtual-care.

Williams, E. (2020, March 6). Empowering care teams with new tools in Microsoft 365. Microsoft 365. Retrieving from https://www. microsoft.com/en-us/microsoft-365/blog/2020/03/06/empowering -care-teams-with-new-tools-in-microsoft-365/

Zoom (2020). Zoom Video Communications, Inc. Retrieved from https ://zoom.us/healthcare.

Zhu, N., Zhang, D., Wang, W., Li, X., Yang, B., Song, J., et al. (2019). A novel coronavirus from patients with pneumonia in China. New England Journal of Medicine, 382(8), 727-733. https://doi. org/10.1056/NEJMoa2001017.

Publisher's Note Springer Nature remains neutral with regard to jurisdictional claims in published maps and institutional affiliations. 